\title{
Integrable Families of Hard-Core Particles with Unequal Masses in a One-Dimensional Harmonic Trap
}

\author{
N. L. Harshman, ${ }^{1,2, *}$ Maxim Olshanii, ${ }^{3}$ A. S. Dehkharghani, ${ }^{2}$ A. G. Volosniev, ${ }^{4}$ Steven Glenn Jackson, ${ }^{5}$ and N. T. Zinner ${ }^{2}$ \\ ${ }^{1}$ Department of Physics, American University, 4400 Massachusetts Avenue NW, \\ Washington, D.C. 20016, USA \\ ${ }^{2}$ Department of Physics and Astronomy, Aarhus University, DK-8000 Aarhus C, Denmark \\ ${ }^{3}$ Department of Physics, University of Massachusetts Boston, Boston, Massachusetts 02125, USA \\ ${ }^{4}$ Institut für Kernphysik, Technische Universität Darmstadt, 64289 Darmstadt, Germany \\ ${ }^{5}$ Department of Mathematics, University of Massachusetts Boston, Boston, Massachusetts 02125, USA
}

(Received 19 April 2017; revised manuscript received 20 June 2017; published 4 October 2017)

\begin{abstract}
We show that the dynamics of particles in a one-dimensional harmonic trap with hard-core interactions can be solvable for certain arrangements of unequal masses. For any number of particles, there exist two families of unequal mass particles that have integrable dynamics, and there are additional exceptional cases for three, four, and five particles. The integrable mass families are classified by Coxeter reflection groups and the corresponding solutions are Bethe-ansatz-like superpositions of hyperspherical harmonics in the relative hyperangular coordinates that are then restricted to sectors of fixed particle order. We also provide evidence for superintegrability of these Coxeter mass families and conjecture maximal superintegrability.
\end{abstract}

DOI: 10.1103/PhysRevX.7.041001

\section{INTRODUCTION}

The complexity of interacting quantum systems can be partially tamed by extrapolating from solvable models, especially in one dimension [1-3]. Prominent examples include the Lieb-Liniger model with zero-range contact interactions in free space [4], the Tonks-Girardeau gas with hard-core contact interactions [5], the Calogero-Moser (CM) model with inverse square interactions either free or in a harmonic trap [6,7], and the extended family of Calogero-Sutherland-Moser (CSM) models [8]. Such models provide insights about the dynamics and thermodynamics of few-body and many-body physics, and they are proving grounds for inquiry into the nature of integrability, solvability, and chaos.

Interest in one-dimensional models has surged because of experiments with ultracold atoms trapped in tight wave guides with interactions controlled by Feshbach and confinement-induced resonances $[9,10]$. These systems are well described by a one-dimensional model with contact interactions [11]. Dynamical effects predicted by this model like delayed thermalization due to integrability at the hard-core limit have been observed [12]. Controllable dynamics and extended coherence times, possibly combined with internal degrees of freedom (d.o.f.) like spin or

\footnotetext{
*harshman@american.edu
}

Published by the American Physical Society under the terms of the Creative Commons Attribution 4.0 International license. Further distribution of this work must maintain attribution to the author(s) and the published article's title, journal citation, and DOI.
Subject Areas: Atomic and Molecular Physics, Quantum Physics hyperfine structure, make such atomic systems suitable for exploring fundamental few-body and many-body quantum physics $[13,14]$ as well as for applications in quantum technologies [15-17].

However, the famous models mentioned above primarily consider equal-mass particles [18]. This article analyzes one-dimensional particles with different masses (but the same frequencies) in a harmonic trap with hard-core contact interactions. Our analysis shows that for particles with certain masses in a certain order, the mass-imbalanced hard-core system is integrable. Conversely, we provide numerical evidence that for other masses, or even the same masses but in a different order, the dynamics are quantum ergodic. Both of these limits possess potentially observable signatures in the energy level statistics [20,21], particle correlations [22], and thermalization dynamics [23,24]. Measurements probing the relationship between ergodicity and entanglement in a closed quantum system have recently been performed in superconducting qubits [25], showing the power for controllable quantum systems to test nonequilibrium thermodynamics.

The possibility for experimental implementation of mass-imbalanced atomic systems has driven multiple recent analyses. For general masses with contact interactions in an equal-frequency harmonic trap there are no exact solutions, so most previous approaches have relied on approximation schemes and numerical methods [26-33]. In this article, we show that for hard-core interactions there exist families of unequal masses for which there are exact solutions for the ground state and all excited states. This extends results first derived for hard-core interactions in free space [34-36]. Exact solutions for hard-core 
interactions form the basis for approximation schemes for strongly interacting systems, providing a valuable benchmark for testing numerical methods [37-39]. This possibility is of special importance for mass-imbalanced systems where $a b$ initio calculations for strong interaction are especially challenging [40].

We derive the criteria for which sets of imbalanced masses are solvable and integrable using a geometrical approach. In the hard-core limit, configuration space is sectioned into $N$ ! disconnected sectors, one for each order of particles. After separating out the center-of-mass and relative hyperradial d.o.f., the dynamics in each ordering sector of relative angular configuration space can be mapped onto hard-wall quantum billiards on a $(N-2)$ dimensional sphere. The domain is a simplex whose shape depends on the mass ratios. For three particles, the six ordering sectors are just arcs of a circles and every set of masses is therefore integrable by separation of variables. For four particles, the 24 ordering sectors are spherical triangles. Quantum billiards in a general spherical triangle cannot be solved by separability, nor can higherdimensional generalizations to $(N-2)$-simplexes on $(N-2)$-spheres. However, when a particular ordering sector tiles the sphere under reflections, then the problem is exactly solvable using something like the method of images. The possible spherical tilings are classified using Coxeter groups. Described in more detail below, Coxeter groups are point symmetry groups generated by reflections. They were originally developed for the purpose of analyzing symmetric polytopes [41]. In hard-core contact interaction models, the $(N-1)$-dimensional hyperplanes where two particles coincide define planes of reflection. If the particle masses are correct, then these coincidence reflection planes generate a Coxeter group.

This logic can be reversed: we show that for every finite, connected, nonbranching Coxeter group with rank $r$ there is a one-parameter family of masses for which the dynamics of $r+1$ ordered particles is integrable. These models are nontrivial when $N>3$; we focus on the case $N=4$, where there are three families of solvable masses and the symmetries in relative configuration space are the same as the Platonic solids. We give special attention to the exceptional Coxeter group $\mathrm{H}_{3}$ of icosahedral symmetries, and therefore this work is closely related to CSM models based on exceptional reflection groups [42-44]. The role of Coxeter groups in providing integrability criteria for this model is perhaps not surprising because they have previously played an important role in the theory of classical and quantum dynamical systems. For example, extensions of the CSM model have a closely related classification scheme [8], and so do Gaudin models [3].

Besides applications to mass-imbalanced ultracold atomic gases, a motivating interest in this model is that it sits at the intersection of related notions of integrability and solvability. For sectors with Coxeter tiling symmetry, the energies can be calculated algebraically and all excited states can be expressed as orthogonal polynomials times the ground state; this property is called exact solvability $[45,46]$. These solutions are constructed by Betheansatz-like superpositions, not of plane waves, but of spherical (or hyperspherical) harmonics. The conditions on the masses can be seen as the requirement that the scattering is nondiffractive, i.e., integrable in the Betheansatz sense [2,47-49]. On the other hand, we provide evidence that these models are also integrable in the classical, Liouvillian sense. Classically, Liouvillian integrability means there are $N$ functionally independent invariant operators in involution that act continuously on $2 N$-dimensional phase space. The quantum versions of these operators commute with each other and therefore all states can be characterized by the spectrum of this set of operators (for a discussion of ambiguities in defining integrability in quantum systems, see Ref. [50]). As an example, we construct these operators for the four-particle case of $\mathrm{H}_{3}$. Further, we conjecture that these solvable models are superintegrable, meaning they have more integrals of motion than d.o.f., and even maximally superintegrable with $2 N-1$ integrals of motion (for a more complete discussion, see Ref. [51]). Superintegrable systems can have a rich mathematical structure, like multiseparability and exact solvability. Further, perturbations from superintegrable models sometimes remain integrable. For example, the unitary (hard-core) limit of equal-mass particles in a harmonic trap with contact interactions is a maximally superintegrable system isomorphic to one limit of the $\mathrm{CM}$ model $[7,52,53]$. In the near-unitary limit, defined as a first-order perturbation from the hard-core limit, maximal superintegrability is broken. However, the system still retains enough symmetry in the near-unitary limit that it can be mapped onto a spin-chain model $[37,38]$. At the other extreme from maximal superintegrability, the Coxeter group criteria could be used to identify massimbalanced systems where quantum ergodic dynamics should be expected. In the experimental outlook, we discuss the connections to quantum billiards and the consequences of integrability for thermalization in ultracold atomic gases.

\section{MODEL AND SYMMETRY}

We consider the $N$-particle Hamiltonian with contact interactions. All particles are harmonically trapped with the same frequency. Written in terms of the particle coordinates $\mathbf{x}=\left(x_{1}, x_{2}, \ldots, x_{N}\right)$, the Hamiltonian has the form

$H=\sum_{i=1}^{N}\left(\frac{-\hbar^{2}}{2 m_{i}} \frac{\partial^{2}}{\partial x_{i}^{2}}+\frac{1}{2} m_{i} \omega^{2} x_{i}^{2}\right)+g \sum_{i<j} \delta\left(x_{i}-x_{j}\right)$.

In the limit $g \rightarrow \infty$ of hard-core contact interactions, the order of the particles is a dynamical invariant [39]. 
Configuration space is divided into $N$ ! ordering sectors by $N(N-1) / 2$ impenetrable coincidence hyperplanes, one for each pair of particles. The order $x_{p_{1}} \leq x_{p_{2}} \leq \cdots \leq x_{p_{N}}$ is labeled by a permutation $p=\left\{p_{1}, p_{2}, \ldots, p_{N}\right\}$, or more briefly $p=p_{1} p_{2} \cdots p_{N}$. We denote by $X_{i j}$ the coincidence hyperplane defined by $x_{i}-x_{j}=0$.

In Appendix A, we show that solving for the eigenstates of Eq. (1) in a particular ordering sector $p$ is equivalent to solving for the motion of a free quantum particle confined to an $(N-2)$-sphere and trapped inside an angular sector $\Omega_{p}$ bounded by $(N-1)$ hard walls. We establish this equivalence by making a mass-dependent transformation $T$ of the position coordinates $\mathbf{z}=T \mathbf{x}$, and then separating out the scaled center of mass $z_{N}$ and the scaled relative hyperradius $\rho$ :

$$
z_{N}=\sqrt{\frac{\omega}{\hbar M}} \sum_{i=1}^{N} m_{i} x_{i} \quad \text { and } \quad \rho^{2}=\sum_{j=1}^{N-1} z_{j}^{2},
$$

where $M$ is the total mass. The remaining relative coordinates are the $(N-2)$ hyperangles $\left\{\phi, \theta_{1}, \ldots, \theta_{N-3}\right\}$ that cover the sphere $\mathcal{S}^{N-2}$. The transformation to these coordinates gives the harmonic potential a spherically symmetric form, but the coincidence hyperplanes, now transformed to $Z_{i j}=T\left(X_{i j}\right)$, break that symmetry. For later convenience, we denote by $\hat{\gamma}_{i j}$ the unit normal vector to the $Z_{i j}$ coincidence hyperplane.

The specific angular ordering sector $\Omega_{p}$ is bounded by the intersection of the sphere with the $(N-1)$ coincidence hyperplanes $Z_{p_{1} p_{2}}, Z_{p_{2} p_{3}}, \ldots, Z_{p_{N-1} p_{N}}$. Each sector $\Omega_{p}$ has $(N-2)$ angles $\omega_{i j k}$ of the form

$$
\omega_{i j k}=\arctan \left(\sqrt{\frac{m_{j}\left(m_{i}+m_{j}+m_{k}\right)}{m_{i} m_{k}}}\right) .
$$

corresponding to the intersections of coincidence planes $Z_{i j}$ and $Z_{j k}$ that share a particle, and $(N-3)(N-2) / 2$ angles of $\omega_{i j, k l}=\pi / 2$ for the intersections of coincidence planes $Z_{i j}$ and $Z_{k l}$ that do not share a particle. For four particles, each ordering sector $\Omega_{p}=\Omega_{i j k l}$ is a spherical triangle bounded by three great circles; see Fig. 1 .

Solving the (hyper)spherical Helmholtz equation on an angular sector $\Omega_{p}$ with Dirichlet boundary conditions is an example of quantum billiards. The problem of quantum and classical billiards in planar triangles is well studied [54-62], and the integrability and solvability of the dynamics depends critically on the domain shape of the billiards. For example, the only three triangular billiards in a plane that have classically integrable dynamics are the three triangles with distinguishable sides that tile the plane under reflections, without gaps or overlaps (see footnote 3 of Ref. [34]). This serves as our guide for the following result for spherical quantum billiards. The dynamics in an

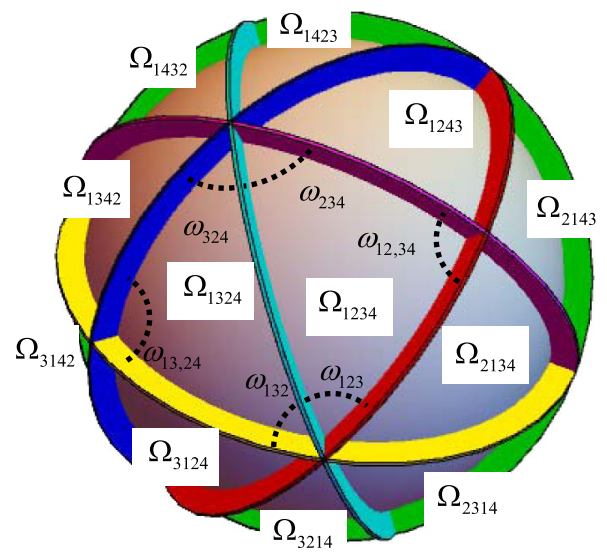

FIG. 1. The relative configuration space for four particles in the simplest case when all masses are the same, an example of the one-parameter mass family of the Coxeter group $A_{3}$. The gray sphere represents an equipotential of the harmonic trap in the mass-normalized coordinates $\left(z_{1}, z_{2}, z_{3}\right)$. The six colored disks that intersect the plane represent the coincidence planes $Z_{12}$ (red), $Z_{13}$ (yellow), $Z_{14}$ (green), $Z_{23}$ (cyan), $Z_{24}$ (blue), $Z_{34}$ (magenta). Twelve sectors are visible and are labeled by $\Omega_{p}$, where $p$ is the order of the four particles. For two sectors $\Omega_{1234}$ and $\Omega_{1324}$, the three angles are also labeled.

angular sector $\Omega_{p}$ is integrable and exactly solvable when the following are satisfied.

(i) The sector $\Omega_{p}$ tiles the $(N-2)$-sphere under reflections across its boundaries. The tiling covers the sphere with no gaps or overlaps and distinguishable sides. In other words, the $(N-2)(N-1) / 2$ angles of a sector $\omega_{i j k}$ and $\omega_{i j, k l}$ define a spherical kaleidoscope.

(ii) The $(N-1)$ reflections across the bounding hyperplanes $Z_{p_{1} p_{2}}, Z_{p_{2} p_{3}}, \ldots, Z_{p_{N-1} p_{N}}$ generate a finite Coxeter reflection group. The $(N-1)$ reflection normals $\hat{\gamma}_{p_{1} p_{2}}, \hat{\gamma}_{p_{2} p_{3}}, \ldots, \hat{\gamma}_{p_{N-1} p_{N}}$ are the simple roots of the Coxeter group.

All finite reflection groups (in all dimensions) were classified by Coxeter [41,63]. Abstractly, a Coxeter group of rank $m$ is a finite group generated by $m$ reflections, where a reflection is a group element that squares to the identity. Every point symmetry group in $m$ dimensions is either a Coxeter group or a subgroup of a Coxeter group of rank $m$. For example, the three-dimensional point groups familiar from chemical and solid-state physics are all subgroups of the Coxeter groups $A_{3}$ (tetrahedral symmetry), $C_{3}$ (cubic symmetry), and $\mathrm{H}_{3}$ (icosahedral symmetry), or they are subgroups of products of lower-rank Coxeter groups.

The structure of the reflection group can be encoded by the Coxeter diagram, which can be branching or nonbranching and connected or not connected. There is a family of $N$ masses that determines a "good" sector for every nonbranching and connected Coxeter reflection group with rank $N-1$. These groups are listed in Table I. Only the nonbranching Coxeter groups are relevant, because in one dimension each pair can have at most 
TABLE I. Connected, nonbranching, finite Coxeter reflection groups. For $N$ particles, each rank $m=N-1$ Coxeter group defines a one-parameter family of masses for which the system is exactly solvable. For each group $G_{m}$, the following data are provided [64]: the Coxeter bracket $\left[q_{1}, \ldots, q_{m}\right]$ from which one determines the angles of the integrable sector; the number of reflections $\lambda_{0}$ in the group which determines the relative angular momentum of the ground-state solution; and the order $G$ of the group which gives the number of integrable sectors required to tile the sphere. Note that there are two series of groups $A_{m}$ and $C_{m}$ that provide integrable mass families for any number of particles.

\begin{tabular}{|c|c|c|c|c|}
\hline$N$ & $A$ series & $C$ series & $H$ type & Others \\
\hline 3 & $\begin{array}{c}A_{2} \equiv I_{2}(3) \\
{[3]} \\
\lambda_{0}=3 \\
G=6\end{array}$ & $\begin{array}{c}C_{2} \equiv I_{2}(4) \\
{[4]} \\
\lambda_{0}=4 \\
G=8\end{array}$ & $\begin{array}{c}H_{2} \equiv I_{2}(5) \\
{[5]} \\
\lambda_{0}=5 \\
G=10\end{array}$ & $\begin{array}{c}I_{2}(q) \\
{[q]} \\
\lambda_{0}=q \\
G=2 q\end{array}$ \\
\hline 4 & $\begin{array}{c}A_{3} \\
{[3,3]} \\
\lambda_{0}=6 \\
G=24\end{array}$ & $\begin{array}{c}C_{3} \\
{[4,3]} \\
\lambda_{0}=9 \\
G=48\end{array}$ & $\begin{array}{c}H_{3} \\
{[5,3]} \\
\lambda_{0}=15 \\
G=120\end{array}$ & \\
\hline 5 & $\begin{array}{c}A_{4} \\
{[3,3,3]} \\
\lambda_{0}=10 \\
G=120\end{array}$ & $\begin{array}{c}C_{4} \\
{[4,3,3]} \\
\lambda_{0}=16 \\
G=384\end{array}$ & $\begin{array}{c}H_{4} \\
{[5,3,3]} \\
\lambda_{0}=60 \\
G=14400\end{array}$ & $\begin{array}{c}F_{4} \\
{[3,4,3]} \\
\lambda_{0}=24 \\
G=1152\end{array}$ \\
\hline$\geq 6$ & $\begin{array}{c}A_{N-1} \\
{\left[3^{N-1}\right]} \\
\lambda_{0}=[N(N-1) / 2] \\
G=(N-1) !\end{array}$ & $\begin{array}{c}C_{N-1} \\
{\left[4,3^{N-2}\right]} \\
\lambda_{0}=(N-1)^{2} \\
G=2^{N} N !\end{array}$ & & \\
\hline
\end{tabular}

two adjacent pairs. Geometrically, this enforces that the coincidence planes of nonadjacent pairs like $Z_{i j}$ and $Z_{k l}$ are orthogonal, $\omega_{i j, k l}=\pi / 2$. We focus our attention on the connected Coxeter groups because they are relevant when all masses are finite. Disconnected graphs realize limiting cases of extreme mass imbalances. For example, for four particles, if the first or fourth particle is much more massive, i.e., like the "Born-Oppenheimer" case of Ref. [26], then the reducible Coxeter groups like $I_{2}(q) \times A_{1}$ could be employed.

For each group in Table I, there exists a one-parameter family of mass sequences for which there are integrable sectors. The bracket notation for the Coxeter group $\left[q_{1}, q_{2}, \ldots, q_{N-2}\right]$ determines the sector angle by Eq. (3), where $\omega_{i(i+1)(i+2)}=\pi / q_{i}$. In Fig. 2, we show the integrable mass spectra for the three four-particle families $A_{3}, C_{3}$, and $H_{3}$. This can be reversed: given a set of $N$ masses in a particular order, one could check how close the sector angles derived from the masses come to the angles $\pi / q_{1}, \ldots, \pi / q_{N-2}$ that define a rank $N-1$ Coxeter group.

For $N$ particles that define a good sector, one that tiles the $(N-2)$-sphere, the generators of the Coxeter group can be chosen as the $m=N-1$ reflections $R_{i j}$ across the boundary hyperplanes $Z_{i j}$ of the sector $p=12 \ldots N$. For $N=4$, the Coxeter groups are rank $m=3$ and are generated by
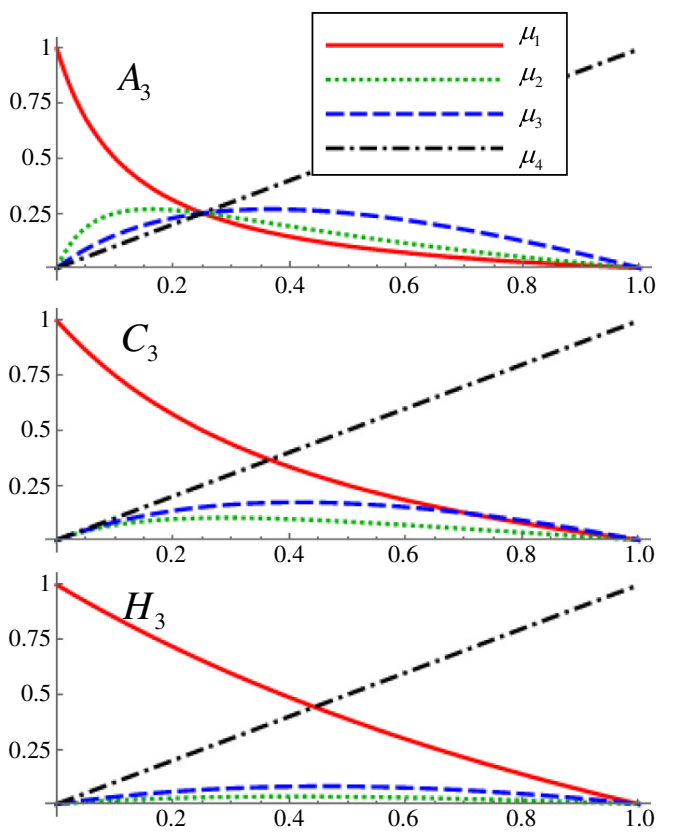

FIG. 2. The integrable mass families for four particles with the Coxeter symmetries $A_{3}, C_{3}$, and $H_{3}$. The mass fractions $\mu_{i}=$ $m_{i} / M$ are plotted versus $\mu_{4}$ (legend is in the top graph). The case where all masses are the same $\mu_{i}=1 / 4$ is in mass family $A_{3}$. Mass family $C_{3}$ includes two cases where two finite masses are the same, and $\mathrm{H}_{3}$ includes one.

$R_{12}, R_{23}$, and $R_{34}$. All three generators square to the identity, and the relations

$$
\left(R_{12} R_{34}\right)^{2}=\left(R_{34} R_{23}\right)^{3}=\left(R_{23} R_{12}\right)^{q}=1
$$

hold for $q=3, q=4$, and $q=5$ for $A_{3}, C_{3}$, and $H_{3}$, respectively. Generally, within each Coxeter group $G_{m}$, there is a conjugacy class $K \subset G_{m}$ of all reflections $R \in G_{m}$. We denote the number of reflection planes (and the order of $K$ ) by $\lambda_{0}$ and denote the normals to these planes by $\hat{\gamma}(R)$, but remember that only $N-1$ of these planes and normals are "real"; i.e., they correspond to the actual coincidence planes and normals. See Fig. 3.

Note that for the Coxeter groups in the $A$ series, $C$ series, and the exceptional group $F_{4}$, the sector angles are all $\pi / 2$, $\pi / 3$, or $\pi / 4$. Inspecting Eq. (3), we see that for these cases the masses are all rational fractions of each other. For example, for the group $C_{3}$ there are a countably infinite number of rational mass sequences that give integrable sectors. The four with the lowest rational denominators are given by $(3 m, m, 2 m, 6 m),(10 m, 2 m, 3 m, 5 m),(12 m, 3 m, 5 m, 10 m)$, and $(56 m, 7 m, 9 m, 12 m)$. As we discuss below, this allows the possibility of building integrable systems out of clusters of particles with the same mass. 

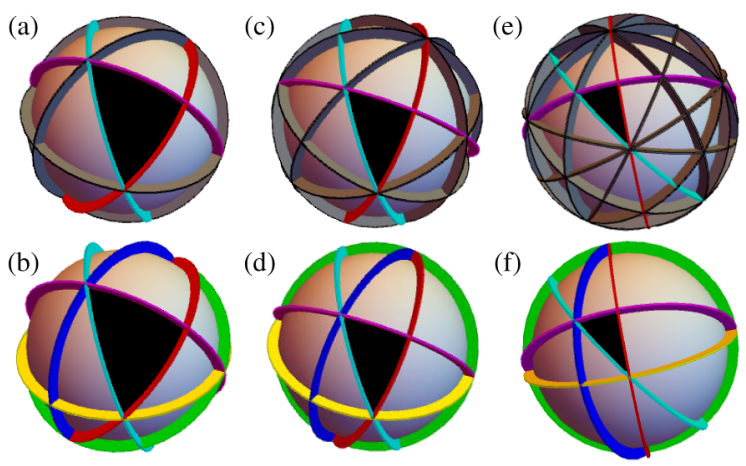

FIG. 3. The top row [(a),(c),(e)] depicts the arrangement of the $\lambda_{0}$ reflection planes (gray and colored disks) and the tiling of sphere into $G$ spherical triangle sectors for $A_{3}, C_{3}$, and $H_{3}$, respectively. The bottom row $[(\mathrm{b}),(\mathrm{d}),(\mathrm{f})]$ shows the coincidence planes (colored disks) for specific, nonsymmetric choices of masses within the mass families for $A_{3}, C_{3}$, and $H_{3}$, respectively. The disk colors are the same as in Fig. 1. The black spherical triangle tiles the sphere in the top figure and it is similar to the integrable sector $\Omega_{1234}$ in the bottom figure. This sector is bounded by the planes $Z_{12}$ (red), $Z_{23}$ (cyan), $Z_{34}$ (magenta), which are the generating planes for the Coxeter symmetry.

\section{EXACT SOLVABILITY AND BETHE-ANSATZ INTEGRABILITY}

For each Coxeter group, there is therefore a oneparameter family of masses such that the complete spectrum of energy eigenstates can be exactly solved in the ordering sector $\Omega_{1 \ldots N}$ and its inverted sector $\Omega_{N \ldots 1}$. The ground state in each of these "Coxeter sectors" is nondegenerate and its hyperangular wave function can be expressed as

$$
\Upsilon_{\lambda_{0}}(\hat{\mathbf{z}})=N_{\lambda_{0}} \prod_{R \in K}(\hat{\gamma}(R) \cdot \hat{\mathbf{z}})
$$

where $\hat{\mathbf{z}}=\left(z_{1}, \ldots, z_{N-1}\right) / \rho$ is a unit vector expressed in hyperspherical coordinates and $N_{\lambda_{0}}$ is a normalizing factor. For all $R \in K$, the function from Eq. (5) is reflection antisymmetric, $\Upsilon_{\lambda_{0}}(R \hat{\mathbf{z}})=-\Upsilon_{\lambda_{0}}(\hat{\mathbf{z}})$, and therefore vanishes on all reflections planes, including the coincidence planes. Note that $\rho^{\lambda_{0}} \Upsilon_{\lambda_{0}}(\hat{\mathbf{z}})$ is the lowest degree anti-invariant polynomial of the corresponding group [63]. The function from Eq. (5) is defined on the entire sphere, but its restriction to the ordering sectors $\Omega_{1 \cdots N}$ or $\Omega_{N \cdots 1}$ provides that sector's ground state with energy $\hbar \omega\left(\lambda_{0}+N / 2\right)$. Exploiting separability, a tower of states are laddered from the ground-state manifold with energies $\hbar \omega\left(n+2 \nu+\lambda_{0}+\right.$ $N / 2$ ), where $n$ is the center-of-mass excitation and $\nu$ is the relative hyperradial excitation [see Appendix A].

For $N$ equal masses, the Coxeter group is $A_{N-1}$ and the ground state corresponds to the lowest-energy fermionic state in a harmonic trap restricted to a sector (à la Girardeau) as expected $[65,66]$. This equal-mass solution can also be seen as the limiting case of the ground state of the Calogero-Moser model with inverse-square interactions in a harmonic trap with zero coupling constant $[67,68]$. However, unlike the equal-mass solutions, the nonequalmass solutions cannot be considered as restrictions of fermionic solutions to a single sector.

In addition to the ground state Eq. (5), the excited state relative hyperangular wave functions in a Coxeter sector are also constructed using a Bethe-ansatz-like superposition of hyperspherical harmonics. Hyperspherical harmonics are homogeneous polynomials in $\hat{z}_{i}$ that are eigenstates of the relative angular momentum $L_{\text {rel }}^{2}$ with eigenvalue $\lambda(\lambda+N-3)[69,70]$. The method takes advantage of the fact that reflections $R_{i j}$ commute with $L_{\text {rel }}^{2}$, or in other words, the Coxeter group of rank $(N-1)$ is a subgroup of the orthogonal transformations $\mathrm{O}(N-1)$ [see Appendix B]. Like Eq. (5), excited solutions are first constructed over the whole sphere, and then restricted to the Coxeter sectors. By construction, the excited states are antisymmetric with respect to reflections in the Coxeter group. Not all values $\lambda>\lambda_{0}$ for the relative angular momentum support such solutions. For the three groups $A_{3}, C_{3}$, and $H_{3}$, the allowed spectra of $\lambda$ are

$$
\begin{aligned}
& A_{3}: \lambda=6+3 n_{1}+4 n_{2}, \\
& C_{3}: \lambda=9+4 n_{1}+6 n_{2}, \\
& H_{3}: \lambda=15+6 n_{1}+10 n_{2} .
\end{aligned}
$$

In each case, the first number in the sum is $\lambda_{0}$, the relative angular momentum of the ground state and the number of reflections in the Coxeter group. Then the non-negative integers $n_{1}$ and $n_{2}$ label the excited states. Degeneracies in the hyperangular d.o.f. arise when multiple pairs of integers provide the same $\lambda$, and the pattern of degeneracies matches the prediction of Weyl's law for a spherical triangle (see below). The series of positive integers $3 n_{1}+4 n_{2}$, $4 n_{1}+6 n_{2}$, and $6 n_{1}+10 n_{2}$ in Eq. (6) corresponds precisely to the orders of homogeneous polynomials that have definite relative angular momentum and are symmetric under the action of reflections in the groups $A_{3}, C_{3}$, and $H_{3}$, respectively [63]. Incorporating the center-of-mass and hyperradial d.o.f., all energy eigenstates are uniquely identified by four quantum numbers: $\left\{n, \nu, n_{1}, n_{2}\right\}$.

For $N \geq 4$ and general masses, or for Coxeter masses but in an arbitrary order, we believe the dynamics within sectors are not integrable in any sense. In the case of $H_{3}$, we provide evidence by numerically solving the spherical Laplacian for Coxeter masses in all sectors. We use the following method [31]: Each spherical triangle is flattened into an isosceles right triangle. The flattening coordinate transformation distorts the spherical Laplacian into a new operator whose spectrum must be solved inside the triangle with hard-wall boundary conditions. The spectrum is found by diagonalizing this transformed 

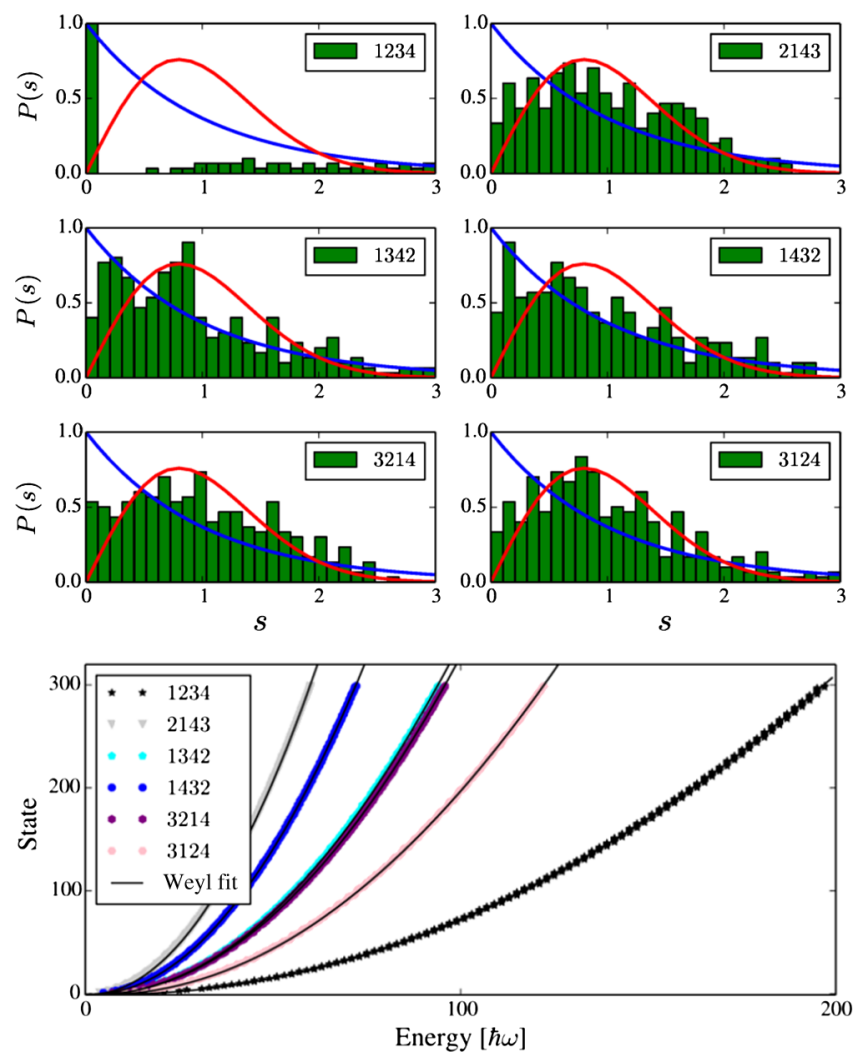

FIG. 4. Unfolded spectrum statistics for $\mathrm{H}_{3}$ Coxeter masses with mass fractions $\mu_{1}=\mu_{4}=0.44279, \mu_{2}=0.03381$, and $\mu_{3}=$ 0.08061 . There are only six different sectors because of two equal masses $m_{1}=m_{4}$ and because $\Omega_{p_{1} p_{2} p_{3} p_{4}}$ is congruent to $\Omega_{p_{4} p_{3} p_{2} p_{1}}$ by inversion. The variable $s$ is the normalized unfolded energy level difference [20]. The integrable sector $\Omega_{1234}$ is depicted in the top left graph and agrees with the prediction from Eq. (6c). The blue lines depict the Poissonian statistics expected for an integrable system; the red lines are Wigner-Dyson distribution derived from random matrix theory expected for quantum ergodic systems with time-reversal symmetric Hamiltonians. The bottom graph shows the quality of Weyl's law Eq. (7) in the integrable sector $\Omega_{1234}$ as well as the nonintegrable sectors.

Laplacian in a basis of exact solutions for the right triangle. Details about this procedure and its convergence are described in Appendix C.

The level spacing statistics (after the standard unfolding [20]) for a set of four particles with $H_{3}$ mass ratios are depicted in Fig. 4. The first sector depicted is the integrable sector, whose numerical solution agrees with the prediction of Eq. (6c), the other five sectors are for the same masses arranged in other orders. For integrable sectors, the unfolded energy level statistics are expected to follow a Poissonian distribution. In our case, within the Coxeter sector the extra degeneracies of the system due to superintegrability distort the distribution so that it is peaked even more strongly at zero-energy gap [21]. What we demonstrate in Fig. 4 is that even for Coxeter mass families, the incorrectly ordered sectors show numerical evidence for quantum ergodicity in the form of Wigner-Dyson distributions for their eigenvalues.

Our numerical results for the spacing statistics open questions about the transition from integrability to ergodicity. We perform numerical simulations on a variety of integrable mass families. While the characteristics of the Coxeter sectors remains stable, the other sectors sometimes look closer or farther from Wigner-Dyson distributions, as is already visible in Fig. 4. We investigate several possibilities for these intermediate distributions, such as integrable subclusters, but we have not arrived at any conclusive results. We also investigate small random deviations from integrable mass sectors of the order of $5 \%$. For this scale of deviation, the formerly integrable sectors still look far from Wigner-Dyson, but closer to Poissonian than the energy level statistics for exact Coxeter masses. Understanding the ragged edge between integrability and ergodicity using this model seems to be a productive avenue for future investigation.

To demonstrate that we find all the spectrum from this procedure, we compare our results for $N(\tilde{E})$, the total number of energy eigenvalues below scaled energy $\tilde{E}$, to the prediction of Weyl's law [71] for a sphere:

$$
N(\tilde{E})=\frac{A}{4 \pi} \tilde{E}-\frac{\ell}{4 \pi} \sqrt{\tilde{E}},
$$

where $\tilde{E}=\left(2 m R^{2} / \hbar^{2}\right) E$ and $R$ and $m$ are arbitrary length and mass parameters constrained by $\hbar \omega=\hbar^{2} /\left(2 m R^{2}\right)$. The second term is the correction due to the Dirichlet boundary conditions proportional to the boundary length $\ell$. The area of the spherical triangle $\Omega_{i j k l}$ is $A / R^{2}=\omega_{i j k}+\omega_{j k l}+$ $\omega_{i j, k l}-\pi$ (Girard's theorem [72]). The perimeter is $\ell / R=\varphi_{i j k}+\varphi_{j k l}+\varphi_{i j, k l}$, where the vertex angles $\left(\varphi_{i j k}\right.$, $\left.\varphi_{j k l}, \varphi_{i j, k l}\right)$ satisfy [73]

$$
\cos \varphi_{i j k}=\frac{\cos \omega_{i j k}+\cos \omega_{j k l} \cos \omega_{i j, k l}}{\sin \omega_{j k l} \sin \omega_{i j, k l}}
$$

and cyclic permutations of $\left(\varphi_{i j k}, \varphi_{j k l}, \varphi_{i j, k l}\right)$ and $\left(\omega_{i j k}, \omega_{j k l}\right.$, $\omega_{i j, k l}$ ). Figure 4 compares numerical solutions to this prediction.

\section{LIOUVILLE INTEGRABILITY AND SUPERINTEGRABILITY}

The separability of the model defined in Eq. (1) provides four functionally independent integrals of the motion for $N \geq 3$ particles with any masses: the center-of-mass Hamiltonian $H_{\text {c.m. }}$., the relative Hamiltonian $H_{\text {rel }}$, the total angular momentum squared $L^{2}$, and the relative angular momentum squared $L_{\mathrm{rel}}^{2}$. Three of these integrals $\left\{H_{\text {c.m. }}, H_{\text {rel }}, L_{\text {rel }}^{2}\right\}$ are in involution, but $L^{2}$ does not commute with them. This set of four integrals of motion is sufficient to prove integrability and superintegrability 
(but not maximal superintegrability) for $N=3$ with any masses. For $N=4$, this is not enough to prove integrability, which requires four integrals in involution, nor is it enough for superintegrability, requiring at least five total conserved quantities, and certainly not enough for maximal superintegrability, requiring seven conserved quantities.

Nonetheless, we conjecture that our model is maximally superintegrable for $N \geq 3$ when in a sector with Coxeter mass ratios. Our evidence is the following: (1) using the method of images [55,74], the classical problem can be shown to support closed orbits indicating maximal superintegrability; (2) the correspondence of our model as a limiting case of certain CSM models which are maximally superintegrable $[52,53]$. The general construction of a set of observables that provide maximal superintegrability for all reflection groups in any number of spatial dimensions requires the methods of algebraic geometry. This ongoing project will be the subject of a future publication.

Let us outline a potential strategy for searching for the missing integrals of motion for four particles using $\mathrm{H}_{3}$ as an example. The key role is played by the invariant polynomials of the group $q_{m}\left(z_{1}, z_{2}, z_{3}\right)$ with order $m$. These are the lowest-order, homogeneous, functionally independent polynomials that remain unchanged under any of the group transformations. They are known and tabulated for all the reflection groups [75]. For $\mathrm{H}_{3}$, there are three invariant polynomials $q_{m}$ with order $m=2,6$, and 10 and (up to a normalization) they are constructed as

$$
q_{m}\left(z_{1}, z_{2}, z_{3}\right)=\sum_{\{\boldsymbol{\sigma}\}}\left(\boldsymbol{\sigma} \cdot\left(z_{1}, z_{2}, z_{3}\right)\right)^{m},
$$

where $\{\boldsymbol{\sigma}\}$ are the set of vectors describing the six fivefold rotation axes of $H_{3}$. From the three polynomials $q_{2}, q_{6}$, and $q_{10}$, we define the three operators $J_{m} \equiv q_{m}\left(L_{12}, L_{23}, L_{31}\right)$. Here, $L_{i j}$ are the components of the vector of the relative angular momentum in the $i j$ plane. Note that $J_{2}$ is proportional to $L_{\text {rel }}^{2}$ and so it does not give an additional integral of motion.

However, the operator $J_{6}$ completes the commuting set $\left\{H_{\text {c.m. }}, H_{\text {rel }}, L_{\text {rel }}^{2}\right\}$ to a Liouvillian set. Since $J_{6}$ commutes with mirror reflections of the $H_{3}$ group, by Schur's lemma it must act as a multiple of the identity on the antisymmetric states. It commutes with the previous three members of the Liouvillian set, and all four can be readily shown to be functionally independent in the classical sense. The fivemember set $\left\{H_{\text {c.m. }}, L^{2}, H_{\text {rel }}, L_{\text {rel }}^{2}, J_{6}\right\}$ now establishes superintegrability for the $H_{3}$ mass family.

This set can be further extended to a maximally superintegrable set using the operator $J_{10}$ and another invariant operator $I_{6}$ defined by

$$
I_{6} \equiv q_{6}\left(a_{1}^{\dagger}, a_{2}^{\dagger}, a_{3}^{\dagger}\right) q_{6}\left(a_{1}, a_{2}, a_{3}\right),
$$

where $a_{j}=\left(-i \partial_{z_{j}}-i z_{j}\right) / \sqrt{2}$ is an annihilation operator for the $j$ th component of the relative motion. The operator
$I_{6}$ naturally commutes with the total Hamiltonian $H$. The resulting seven-member set is (classically) functionally independent and establishes maximal superintegrability for the $\mathrm{H}_{3}$ model. The scheme can readily be generalized to the other two three-dimensional reflection groups, $A_{3}$ and $C_{3}$. However, no ready generalization to higher dimensions exists for the Liouvillian sets, because, a priori, the operators $J_{m}$ do not commute between themselves. Finding Liouvillian sets for higher-dimensional groups is a subject of future work. Identifying and classifying the maximal superintegrablty sets, and ideally connecting them to the known integrals for the Calogero-Moser model $[52,53,76]$, is another ongoing project.

\section{EXPERIMENTAL OUTLOOK}

At the moment, three possible experimental applications of the models we consider in this article can be foreseen. The first possibility is the straightforward idea of finding a collection of atoms that naturally have the right mass ratios and seeking the signatures of integrability in the spectral, coherence, and thermalization properties of the system. Even if the particles are only close to a Coxeter family, our numerical results for the energy spectrum suggest that traces of integrability should still be present. More generally, the Coxeter criteria can be used to measure how far from integrability particular arrangements of imbalanced masses are expected to be, or whether there are integrable subclusters possible within a multispecies ultracold atomic gas.

In the second scheme, if real masses with the correct ratio are not available, the atomic mass is controlled using optical lattices. Given sufficient laser power, the effective mass [77] can be tuned from its "bare" value to almost zero [78]. In particular, the effective mass can be made 3 times greater than its bare counterpart in a lattice of a depth $V_{0}=7 E_{R}$, and 23 times greater for $V_{0}=16 E_{R}$, respectively. Here, $E_{R}=\hbar^{2} k^{2} /(2 m)$ is the so-called recoil energy, $k$ is the wave vector of light that creates the lattice, and $m$ is the bare atomic mass. In both cases, harmonic confinement represents the most natural experimental environment, unlike the box and ring geometries traditionally studied using Bethe-ansatz methods.

In the third scheme, described in more detail in Ref. [36], the role of massive particles is played by bosonic solitons in an atom waveguides [79-81]. The solitons are made of atoms in two alternating internal states where the intraspecies interaction is attractive and the interspecies interaction is repulsive. The goal would be to engineer clusters of atoms whose combined masses satisfy the Coxeter criteria. For example, the clustering pattern $(3 m, m, 2 m, 6 m)$ has $C_{3}$ symmetry. A mixture of ${ }^{7} \mathrm{Li}$ atoms with $m_{F}=-1$ and $m_{F}=0$ in a magnetic field of $855 \mathrm{G}$ constitutes an example [82].

Any implementation of the models considered in our article may constitute an efficient experimental realization of spherical triangular (or higher-dimensional, 
simplex-shaped) quantum billiards [83]. The ergodicity of classical flat triangular billiards is conjectured to strongly depend on the rationality of the billiard angles [59,62]. Numerically, such questions about ergodicity are difficult, requiring long propagation for averages to converge to their infinite time limits. A study of the eigenstate-to-eigenstate variance of the expectation values of observables $[23,84,85]$, which is a faithful quantum analogue of classical deviations from ergodicity (cf. Ref. [86] for a comparison), may provide an efficient alternative to classical long-time averages. Experimentally, one may conjecture an appearance of a memory of initial conditions, if the billiard is not ergodic [87]. The mass mixtures we consider in our paper could constitute a way to study multidimensional classical and quantum hard-wall billiards with continuously tunable geometry, a powerful extension of the existing experimental techniques [88].

\section{ACKNOWLEDGMENTS}

This work was supported by the Aarhus University Research Foundation, the U.S. National Science Foundation Grant No. PHY-1607221, the Office of Naval Research Grant No. N00014-12-1-0400, The Danish Council for Independent Research (Sapere Aude), and the Humboldt Foundation. The authors thank F. F. Bellotti, M.E. S. Andersen, L. Rammelmüller, and F. Werner for useful conversations.

\section{APPENDIX A: COORDINATE TRANSFORMATIONS AND THE MAP TO QUANTUM BILLIARDS}

Here, we establish the map from the model Hamiltonian from Eq. (1) to a free particle in a bounded region on the $(N-2)$-sphere. Much of this is well known [31,89], but we reproduce it here for the readers' convenience and to establish notation.

The equipotentials of Eq. (1) are $N$-dimensional ellipsoids segmented into $N$ ! sectors by $(N-1)$-dimensional hyperplanes $X_{i j}$ defined by the particle coincidences $x_{i}-x_{j}=0$; see Fig. 5. In the limit $g \rightarrow \infty$, these planes are impenetrable. The angle between coincidence hyperplanes $X_{i j}$ and $X_{j k}$ that share a particle is $\pi / 3$ (possible for only $N \geq 3$ ); the angle between hyperplanes $X_{i j}$ and $X_{k l}$ that do no share a particle is $\pi / 2$ (possible for only $N \geq 4$ ).

As a first step, we scale the position coordinates into unitless position variables $y_{i}=\sqrt{m_{i} \omega / \hbar} x_{i}$. Then the Hamiltonian from Eq. (1) becomes

$$
\begin{aligned}
H= & \frac{\hbar \omega}{2} \sum_{i=1}^{N}\left(-\frac{\partial^{2}}{\partial y_{i}^{2}}+y_{i}^{2}\right) \\
& +\sum_{i<j} \tilde{g}_{i j} \delta\left(\sqrt{\frac{\mu_{i j}}{m_{i}}} y_{i}-\sqrt{\frac{\mu_{i j}}{m_{j}}} y_{j}\right),
\end{aligned}
$$
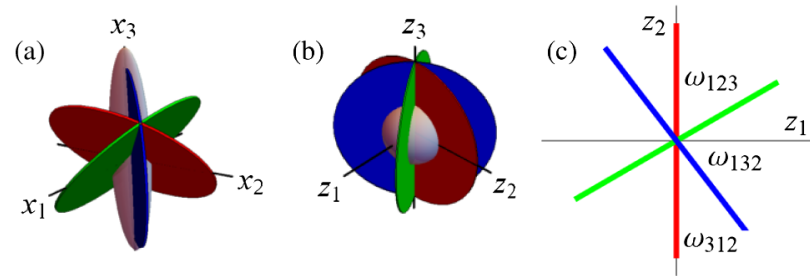

FIG. 5. Coordinate transformation for three particles with the $A_{2}$ masses $m_{1}=1 / 13 M, m_{2}=9 / 52 M$, and $m=3 / 4 M$, where $M$ is the total mass. (a) Configuration space in natural particle positions $\mathbf{x}$. The gray ellipsoid represents an equipotential for the equal frequency (and therefore not equal strength), and the red, green, and blue disks represent the $X_{12}, X_{23}$, and $X_{31}$ coincidence planes, respectively. (b) Configuration space in mass-scaled and rotated coordinates $\mathbf{z}=J \mathbf{x}$, with a spherical equipotential and transformed coincidence planes $Z_{12}, Z_{23}$, and $Z_{31}$. (c) Projection of structure in (b) into relative coordinate $z_{1}-z_{2}$ plane. The sector with angle $\omega_{123}=\pi / 3$ is the Coxeter sector.

where $\mu_{i j}=m_{i} m_{j} /\left(m_{i}+m_{j}\right)$ and $\tilde{g}_{i j}=g_{i j} \sqrt{\mu_{i j} \omega / \hbar}$. This scaling transformation $\mathbf{y}=S \mathbf{x}$ has brought the harmonic potential into a form with $N$-spherical symmetry but at the cost of desymmetrizing the coincidence planes. To describe the geometry, we define the transformed coincidence planes $Y_{i j} \equiv S X_{i j}$ with normals $\hat{\boldsymbol{\beta}}_{i j}$. The contact interaction then has the form

$$
\sum_{i<j} \tilde{g}_{i j} \delta\left(\hat{\boldsymbol{\beta}}_{i j} \cdot \mathbf{y}\right)
$$

The angle $\omega_{i j k}$ between coincidence planes $Y_{i j}$ and $Y_{j k}$ with normals $\hat{\boldsymbol{\beta}}_{i j}$ and $\hat{\boldsymbol{\beta}}_{j k}$ is now

$$
\omega_{i j k}=\arctan \left(\sqrt{\frac{m_{j}\left(m_{i}+m_{j}+m_{k}\right)}{m_{i} m_{k}}}\right) .
$$

Whereas in the equal mass case, $\omega_{i j k}$ is always $\pi / 3$, for three arbitrary masses it can range from $0\left(m_{j}\right.$ much lighter than the other two masses) to $\pi / 2$ ( $m_{j}$ much heavier). The angle $\omega_{i j, k l}$ between coincidence planes $Y_{i j}$ and $Y_{k l}$ that do not share a particle remains $\pi / 2$.

In the limit $g \rightarrow \infty$, the Hamiltonian from Eq. (A1) separates in hyperspherical coordinates with radius $R^{2}=\sum_{i} y_{i}^{2}$. The interaction term Eq. (A2) is proportional to $1 / R$, so it is not separable for finite values of $\tilde{g}_{i j}$, but as $\tilde{g}_{i j} \rightarrow \infty$ there is no distinction between $1 / R$ or $1 / R^{2}$ times the sum of delta functions. So hyperspherical symmetry of Eq. (A1) emerges and there is $\mathrm{SO}(2,1)$ dynamical symmetry in the total hyperradial coordinate $R$ [89]. To develop physical intuition, it is sometimes useful to imagine a single, classical particle bouncing around in this $N$-dimensional landscape. In this mass-rationalized geometry, the classical particle trajectory changes its direction of angular momentum when it bounces off of a coincidence hyperplane $Y_{i j}$, but 
it does not change its magnitude of "angular momentum" in configuration space. However, total angular momentum does not respect the center-of-mass separability and does not commute with the relative Hamiltonian or relative angular momentum (see below), so we do not exploit it here.

Next, we rotate the coordinate system $\mathbf{z}=J \mathbf{y}$ so that the component $z_{N} \equiv Z$ is the scaled center of mass $Z=$ $\sum_{i} y_{i} \sqrt{m_{i} / M}$ and $M$ is the total mass. The orthogonal transformation $J$ with this property is not unique and its selection determines a particular choice for Jacobi relative coordinates $z_{1}$ through $z_{N-1}$. The transformation $J$ also rotates the coincidence planes $Z_{i j} \equiv J Y_{i j}$ and their normals $\hat{\gamma}_{i j} \equiv J \hat{\boldsymbol{\beta}}_{i j}$, but leaves the angles between planes like $\omega_{i j k}$ and $\omega_{i j, k l}$ invariant. Since all the normal vectors $\hat{\gamma}_{i j}$ have zero $Z$ components, the Hamiltonian in $\mathbf{z}$ coordinates separates into $H=H_{\text {c.m. }}+H_{\text {rel }}$, where $H_{\text {c.m. }}$ is the Hamiltonian for a one-dimensional harmonic oscillator in the center-of-mass $Z$ coordinate and the relative Hamiltonian is

$$
H_{\text {rel }}=\frac{\hbar \omega}{2} \sum_{i=1}^{N-1}\left(-\frac{\partial^{2}}{\partial z_{i}^{2}}+z_{i}^{2}\right)+\sum_{i<j} \tilde{g}_{i j} \delta\left(\hat{\gamma}_{i j} \cdot \mathbf{z}\right) .
$$

Finally, we go to hyperspherical coordinates in the relative space, where the relative hyperradius $\rho$ is

$$
\rho^{2}=\sum_{i=1}^{N-1} z_{i}^{2}=\sum_{i=1}^{N} y_{i}^{2}-Z^{2},
$$

and there are $(N-2)$ angles charting the sphere $\mathcal{S}^{N-2}$, conventionally chosen as $\Omega=\left\{\phi, \theta_{1}, \ldots, \theta_{N-3}\right\}$, with $\phi \in$ $[0,2 \pi)$ and $\theta_{i} \in[0, \pi]$. The relative Hamiltonian now becomes

$$
\begin{aligned}
H_{\text {rel }}= & \frac{\hbar \omega}{2}\left[-\frac{1}{\rho^{N-2}} \frac{\partial}{\partial \rho}\left(\rho^{N-2} \frac{\partial}{\partial \rho}\right)-\frac{1}{\rho^{2}} \Delta_{\Omega}+\rho^{2}\right] \\
& +\sum_{i<j} \frac{\tilde{g}_{i j}}{\rho} \delta\left(\hat{\gamma}_{i j} \cdot \hat{z}\right),
\end{aligned}
$$

where $\Delta_{\Omega}$ is the angular part of the Laplacian in relative configuration space. As before, the relative Hamiltonian Eq. (A6) is $\rho-\Omega$ separable in the limit $\tilde{g}_{i j} \rightarrow \infty$. A general energy eigenstate can be separated into a product of center of mass $\zeta(Z)$, relative hyperradial $R(\rho)$, and relative hyperangular $\Upsilon(\Omega)$ functions,

$$
\Phi_{n, \nu, \lambda, \mu}(Z, \rho, \Omega)=\zeta_{n}(Z) R_{\nu \lambda}(\rho) \Upsilon_{\lambda, \mu}(\Omega)
$$

where $n$ is the center-of-mass quantum number, the function $\zeta_{n}(Z)$ is the one-dimensional harmonic oscillator wave function, and $\nu$ is the relative hyperradial quantum number. At this point, $\lambda$ is just derived from the judiciously parametrized relative hyperangular separation constant $\lambda(\lambda+N-3)$ and $\mu$ is just an additional label to distinguish any possible degenerate states for a given $\lambda$. If there was no angular potential $\tilde{g}_{i j}=0$, then $\lambda$ would be a non-negative integer, the eigenfunctions on $\mathcal{S}^{N-2}$ would be the hyperspherical harmonics, and $\mu$ would be a collective index to label degeneracies $[69,70]$. However, since there is an angular potential in Eq. (A6), the hyperangular solutions are unknown and we must explicitly solve for $\lambda$, including any possible degeneracies. Whatever value $\lambda$ takes (including noninteger values), the relative hyperradial function $R_{\nu \lambda}(\rho)$ is the standard solution for the radial factor of an $(N-1)$-dimensional isotropic harmonic oscillator in hyperspherical coordinates [70] with energy $\hbar \omega[2 \nu+\lambda+(N-1) / 2]$ :

$$
R_{\nu \lambda}(\rho)=A_{\nu, \lambda} \rho^{\lambda} L_{\nu}^{\lambda+[(N-3) / 2]}\left(\rho^{2}\right) e^{-\rho^{2} / 2},
$$

where $A_{\nu, \lambda}=\sqrt{2 \nu ! / \Gamma[\nu+\lambda+(N-1) / 2]}$.

We have achieved our desired result: this series of coordinate transformations has reduced solving the $\mathrm{N}$ particle Hamiltonian from Eq. (1) with equal frequencies and infinite-strength contact interactions into solving hardwall quantum billiards in $(N-2)$-simplexes on $(N-2)$ spheres.

\section{APPENDIX B: CONSTRUCTION OF EXACT SOLUTIONS}

Here, we construct the wave functions within the Coxeter sectors, i.e., sectors of the $\mathcal{S}^{N-2}$ hypersphere defined by the relative hyperangular coordinates that have the right shape to tile the sphere under reflection. For convenience, we choose the Coxeter sector to be the ordering sector $\Omega_{12 \ldots N}$ so that it is bounded by the coincidence hyperplanes $Z_{12}$ through $Z_{(N-1) N}$. These $(N-1)$ hyperplanes define the reflections $R_{12}$ though $R_{(N-1) N}$ that generate the Coxeter group.

The Coxeter group $G_{m}$ is generated by $m$ reflections in $m$ dimensions. As such, it can considered as a subgroup of $\mathrm{O}(m)$, orthogonal transformations in $m$ dimensions, and the symmetry of the sphere $\mathcal{S}^{m-1}$. To summarize the method, there is a solution to the Hamiltonian in the Coxeter sectors $\Omega_{12 \ldots N}$ and $\Omega_{N \ldots 21}$ whenever an irreducible representation (irrep) of $G_{N-1}$ that is antisymmetric under all reflections appears in the decomposition of an irrep of $\mathrm{O}(N-1)$. The irreps of $\mathrm{O}(\mathrm{m})$ generally are reducible with respect to the subgroup $G_{m}$. The method of characters can answer the question as to whether an irrep of a subgroup appears in the decomposition of the irreps of the group. When it does exist, the corresponding states can be constructed using projection operators and (in the case of degeneracies) an orthonormalization procedure.

The irreducible representations for $\mathrm{O}(\mathrm{m})$ and their realizations by hyperspherical harmonics are well known [90,91], and so we just summarize a few facts here for the readers' convenience. The subgroup $\mathrm{SO}(m)$ is a Lie group with $m(m-1) / 2$ generators in the Lie algebra. We denote these generators as $L_{i j}$ for $i<j$, with $i, j \in\{1, \ldots, m\}$, 
where $L_{i j}$ generates a rotation in the $i j$ plane. The quadratic Casimir of $\mathrm{SO}(m)$ is the sum of all of these generators squared:

$$
L^{2}=\sum_{\langle i, j\rangle} L_{i j}^{2}
$$

For $\mathrm{SO}(3)$ this is the familiar angular momentum squared operator with eigenvalues $\lambda(\lambda+1)$. The $\mathrm{SO}(3)$ irreps are labeled by $\lambda$ and have degeneracy $2 \lambda+1$. In $m>3$ dimensions, the operator $L^{2}$ is the hyperangular momentum squared operator with eigenvalue $\lambda(\lambda+m-2)$ and an irrep labeled by $\lambda$ has degeneracy [69]

$$
d(\lambda)=\frac{(m+2 \lambda-2)(m+\lambda-3) !}{\lambda !(m-2) !} .
$$

Once the representation of total inversion is chosen, the irreps of $\mathrm{SO}(m)$ also naturally carry a representation of $\mathrm{O}(m)$. For example, inversion is represented in the $\lambda$ irrep by multiplication by $(-1)^{\lambda}$ for $\mathrm{O}(3)$.

To reduce an $\mathrm{O}(\mathrm{m})$ irrep $\lambda$ into the irreps of Coxeter group $G_{m}$, the $G$ elements are sorted into conjugacy classes $K_{i}$ with $k_{i}$ elements. Each irrep $W$ of $G_{m}$ has a unique pattern of characters $\chi^{W}\left(K_{i}\right)$. In particular, we are interested in the $G_{m}$ irrep $W=A$ of all anti-invariant states, meaning $\chi^{A}\left(K_{i}\right)=1$ when $K_{i}$ is a conjugacy class whose elements are an even composition of reflections and $\chi^{A}\left(K_{i}\right)=-1$ when $K_{i}$ is a class composed of odd compositions. Further, each conjugacy class has a character $\chi^{\lambda}\left(K_{i}\right)$ in the $G_{m^{-}}$reducible $\mathrm{O}(m)$ irrep denoted by $\lambda$. When these characters are known, then the number of times the $G_{m}$ irrep $A$ appears in the decomposition of the $\mathrm{O}(m)$ irrep $\lambda$ is [90]

$$
a_{\lambda}=\frac{1}{G} \sum_{K_{i}} k_{i} \chi^{A}\left(K_{i}\right) \chi^{\lambda}\left(K_{i}\right)
$$

Note that $\chi^{A}\left(K_{i}\right)=\left(\chi^{A}\left(K_{i}\right)\right)^{*}$ because Coxeter groups are ambivalent. The number $a_{\lambda}$ is an integer that counts how many solutions there are with relative angular momentum $\lambda$. The projection operator onto the anti-invariant irrep $A$ is given by

$$
P^{A}=\frac{1}{G} \sum_{g \in G_{m}} \chi^{A}(g) D^{\lambda}(g)
$$

where $D^{\lambda}(g)$ is the representation of group element $g$ acting irreducibly on the $d(\lambda)$-dimensional representation space. If $a^{\lambda}=1$, any vector in the $\lambda$ irrep space with a nonzero projection will be proportional to the solution we seek. If $a_{\lambda}>1$, then a set of orthonormal solutions can be found by projecting multiple vectors and then applying GramSchmidt orthogonalization.
TABLE II. Conjugacy classes of $H_{3}$. The first column is the Schönflies notation for the elements in the class $K_{i}$. The second column gives the angle of rotation $\phi_{i}$ of the element realized in $\mathrm{O}$ (3). The third column is whether it is generated by an even or odd number of reflections $\pi_{i}= \pm 1$. All odd elements that are rotations can be considered as rotoreflections, i.e., a rotation followed by a reflection in the plane perpendicular to the rotation axis. The fourth and fifth columns are the order of the elements in the class $K_{i}$ and the number of elements $k_{i}$ in the class, respectively.

\begin{tabular}{lcccc}
\hline \hline Elements & Angle & Even or odd & Order & Number \\
\hline$E$ & 0 & + & 1 & 1 \\
$C_{5}, C_{5}^{4}$ & $2 \pi / 5$ & + & 5 & 12 \\
$C_{5}^{2}, C_{5}^{3}$ & $4 \pi / 5$ & + & 5 & 12 \\
$C_{3}, C_{3}^{2}$ & $2 \pi / 3$ & + & 3 & 20 \\
$C_{2}$ & $\pi$ & + & 2 & 15 \\
$I$ & $\pi$ & - & 2 & 1 \\
$S_{10}, S_{10}^{9}$ & $\pi / 5$ & - & 10 & 12 \\
$S_{10}^{3}, S_{10}^{7}$ & $3 \pi / 5$ & - & 10 & 12 \\
$S_{6}, S_{6}^{5}$ & $\pi / 3$ & - & 6 & 20 \\
$\sigma$ & 0 & - & 2 & 15 \\
\hline \hline
\end{tabular}

As an example, consider the Coxeter group $\mathrm{H}_{3}$. This group has ten conjugacy classes summarized in Table II. The $H_{3}$ character $\chi^{A}\left(K_{i}\right)$ for the anti-invariant irrep is +1 for the five even conjugacy classes and -1 for the five odd classes. The $\mathrm{O}(m)$ character $\chi^{\lambda}\left(K_{i}\right)$ for irrep $\lambda$ is

$$
\chi^{\lambda}\left(K_{i}\right)=\sum_{\mu=-\lambda}^{\lambda} \cos \left(m \phi_{i}\right)\left(\pi_{i}\right)^{\lambda-\mu},
$$

where $\phi_{i}$ is the angle of rotation and $\pi_{i}$ is the reflection parity for the conjugacy class $K_{i}$. Plugging this into Eq. (B1), we find the pattern of degeneracies given in Eq. (6c) of the main text. The same method is used in Ref. [92] to find which $\mathrm{O}(m)$ irreps have symmetric irreps of the spherical triangle groups in their reduction.

To construct the actual states $\Upsilon_{\lambda}(\theta, \phi)$, we use the projection operator Eq. (B2) acting on the spherical harmonics. Instead of explicitly constructing the $(2 \lambda+1) \times$ $(2 \lambda+1)$ unitary matrices $D^{\lambda}(g)$ that act on the spherical harmonics $Y_{\lambda}^{\mu}(\theta, \phi)$ for each of the 120 elements of $H_{3}$, we choose a slightly different method that takes advantage of two facts: (1) the spherical harmonics can be written as polynomials of the relative coordinates, and (2) we already have the $3 \times 3$ matrices $O(g) \in \mathrm{O}(3)$ that represent $H_{3}$ as rotation and reflections.

The first step is to express the spherical harmonics in terms of the relative coordinates $\left(z_{1}, z_{2}, z_{3}\right)$. We work with the real form of the spherical harmonics, defined as

$$
Y_{\lambda \mu}(\theta, \phi)= \begin{cases}\sqrt{2} N_{\lambda \mu} P_{\lambda}^{\mu}(\cos \theta) \cos (\mu \phi) & \mu>0 \\ N_{\lambda 0} P_{\lambda}^{0}(\cos \theta) & \mu=0 \\ \sqrt{2} N_{\lambda \mu} P_{\lambda}^{|\mu|}(\cos \theta) \sin (|\mu| \phi) & \mu<0,\end{cases}
$$


where

$$
N_{\lambda \mu}=\sqrt{\frac{2 \lambda+1}{4 \pi} \frac{(\lambda-|\mu|) !}{(\lambda+|\mu|) !}} .
$$

The real spherical harmonics can be written in terms of the relative coordinates:

$$
Y_{\lambda \mu}(\hat{\mathbf{z}}) \equiv Y_{\lambda \mu}\left[\cos ^{-1}\left(z_{3} / \rho\right), \tan ^{-1}\left(z_{2} / z_{1}\right)\right] .
$$

Noting the relations

$$
\begin{aligned}
\cos (\mu \phi) & =\left(z_{1}^{2}+z_{2}^{2}\right)^{-\mu / 2} \sum_{k=0}^{\mu}\left(\begin{array}{l}
\mu \\
k
\end{array}\right) z_{1}^{\mu-k} z_{2}^{k} \cos \frac{\pi k}{2}, \\
\sin (|\mu| \phi) & =\left(z_{1}^{2}+z_{2}^{2}\right)^{-\mu / 2} \sum_{k=0}^{\mu}\left(\begin{array}{l}
\mu \\
k
\end{array}\right) z_{1}^{\mu-k} z_{2}^{k} \sin \frac{\pi k}{2}, \\
P_{\lambda}^{\mu}(x) & =(-1)^{\mu}\left(z_{1}^{2}+z_{2}^{2}\right)^{\mu / 2} \frac{d^{\mu}}{d\left(z_{3} / \rho\right)^{\mu}} P_{\lambda}\left(z_{3} / \rho\right),
\end{aligned}
$$

we can show that $\rho^{\lambda} Y_{\lambda \mu}(\hat{\mathbf{z}})$ are homogeneous polynomials of order $\lambda$ in $\left(z_{1}, z_{2}, z_{3}\right)$.

The projection Eq. (B2) is applied using the transformation matrix $O(g)$ :

$$
\rho^{\lambda} P^{A} Y_{\lambda \mu}(\hat{\mathbf{z}})=\frac{\rho^{\lambda}}{G} \sum_{g \in G_{m}} \chi^{A}(g) Y_{\lambda \mu}[O(g) \hat{\mathbf{z}}] .
$$

This projection will be zero unless $\lambda$ is in the spectrum given by Eq. (6c) in the main text. Note that it may also be zero for any particular $\mu$, but there must be as many linearly independent polynomials (that also solve the spherical Laplacian) as there are solutions for $n_{1}$ and $n_{2}$ for a given $\lambda$ in Eq. (6c). For $H_{3}$, the first time there are multiple solutions is when $\lambda=45$. Explicit calculation for $\lambda=\lambda_{0}=$ 15 confirms Eq. (5) from the main text for the ground state of $H_{3}$, and we perform the same calculations for the other $N=4$ Coxeter groups.

\section{APPENDIX C: NUMERICAL METHOD FOR SOLVING SPHERICAL TRIANGLE}

Here, we present the numerical method to calculate the energy spectrum for $N=4$ particles with arbitrary masses, which is an extension of the method found in Ref. [31] to spherical triangles. The general strategy is outlined in the main text: here, we provide details about the coordinates, the flattening, and the exact diagonalization we use to construct Fig. 4.

After separation of variables, we must solve for the hyperangular wave function $\Upsilon(\Omega)$ within a sector $\Omega_{p}$. This function must satisfy

$$
\Delta_{\Omega} \Upsilon(\Omega)=\lambda(\lambda+1) \Upsilon(\Omega),
$$

with Dirichlet boundary conditions on the three bounding coincidence planes, $Z_{p_{1} p_{2}}, Z_{p_{2} p_{3}}$, and $Z_{p_{3} p_{4}}$. To solve the problem for arbitrary masses, a particular rotation $J$ in $\mathbf{z}=J S \mathbf{x}$ must be specified. For numerical simplicity, we choose the $H$-type four-body coordinates [93]. Then the rotation $J$ aligns the coordinate plane $Z_{12}$ with the plane $z_{1}=0$ and aligns the coordinate plane $Z_{34}$ with $z_{2}=0$ (see Fig. 1). The other four coincidence planes are given by the following equations:

$$
\begin{aligned}
& Z_{13}: \sqrt{\frac{m_{2}\left(m_{3}+m_{4}\right)}{m_{1} M}} z_{1}-\sqrt{\frac{m_{4}\left(m_{1}+m_{2}\right)}{m_{3} M}} z_{2}+z_{3}=0, \\
& Z_{14}: \sqrt{\frac{m_{2}\left(m_{3}+m_{4}\right)}{m_{1} M}} z_{1}+\sqrt{\frac{m_{3}\left(m_{1}+m_{2}\right)}{m_{4} M}} z_{2}+z_{3}=0, \\
& Z_{23}: \sqrt{\frac{m_{1}\left(m_{3}+m_{4}\right)}{m_{2} M}} z_{1}+\sqrt{\frac{m_{4}\left(m_{1}+m_{2}\right)}{m_{3} M}} z_{2}-z_{3}=0, \\
& Z_{24}: \sqrt{\frac{m_{1}\left(m_{3}+m_{4}\right)}{m_{2} M}} z_{1}-\sqrt{\frac{m_{3}\left(m_{1}+m_{2}\right)}{m_{4} M}} z_{2}-z_{3}=0 .
\end{aligned}
$$

The numerical problem is solved independently in each sector $\Omega_{p_{1} p_{2} p_{3} p_{4}}$ and checked for consistency with the similar sector $\Omega_{p_{4} p_{3} p_{2} p_{1}}$. In the following, as an example, we solve the sector $\Omega_{1342}$, which is limited by $Z_{34}, Z_{13}$, and $Z_{24}$ coincidence planes. We isolate $z_{1}$ in the above equations and introduce (nonstandard) spherical coordinates with $z_{1}=\rho \cos \theta, \quad z_{2}=\rho \sin \theta \cos \phi$, and $z_{3}=$ $\rho \sin \theta \sin \phi$, so that $\cos \theta>0$ in sector $\Omega_{1342}$. Then, substituting spherical coordinates into the coincidence plane equations and dividing by $z_{1}=\rho \cos \theta>0$, we find

$$
\begin{aligned}
\tan \theta \cos \phi & =0, \\
1-a \tan \theta \cos \phi+b \tan \theta \sin \phi & =0, \\
1-c \tan \theta \cos \phi-d \tan \theta \sin \phi & =0,
\end{aligned}
$$

with

$$
\begin{array}{ll}
a=\sqrt{\frac{\left(m_{1}+m_{2}\right) m_{1} m_{4}}{\left(m_{3}+m_{4}\right) m_{3} m_{2}}}, \quad b=\sqrt{\frac{M m_{1}}{\left(m_{3}+m_{4}\right) m_{2}}}, \\
c=\sqrt{\frac{\left(m_{1}+m_{2}\right) m_{2} m_{3}}{\left(m_{3}+m_{4}\right) m_{1} m_{4}}}, \quad d=\sqrt{\frac{M m_{2}}{\left(m_{3}+m_{4}\right) m_{1}}} .
\end{array}
$$

Introducing new coordinates $u=\tan \theta \cos \phi$ and $v=\tan \theta \sin \phi$, the boundary conditions

$$
\begin{aligned}
u & =0, \\
1-a u+b v & =0, \\
1-c u-d v & =0
\end{aligned}
$$

are simple and describe a triangle in flat space. However, the differential operator $\Delta_{\Omega}$ has become more complicated: 


$$
\begin{aligned}
\Delta_{\Omega}= & \left(1+u^{2}+v^{2}\right)\left\{\left(1+u^{2}\right) \frac{\partial^{2}}{\partial u^{2}}+\left(1+v^{2}\right) \frac{\partial^{2}}{\partial v^{2}}\right. \\
& \left.+(2 u v) \frac{\partial^{2}}{\partial u \partial v}+(2 u) \frac{\partial}{\partial u}+(2 v) \frac{\partial}{\partial v}\right\} .
\end{aligned}
$$

Next, we introduce a final coordinate transformation:

$$
\begin{aligned}
& s=\frac{2 d}{(b+d)}(-a u+b v)-\frac{(b-d)}{(b+d)}, \\
& t=\frac{2 b}{(b+d)}(-c u-d v)-\frac{(d-b)}{(b+d)} .
\end{aligned}
$$

This can be inverted as

$$
\begin{aligned}
& u=-\frac{(b+d)}{2(a d+b c)}(s+t), \\
& v=-\frac{a(b+d)}{2 b(a d+b c)}(s+t)+\frac{(b-d)+(b+d) s}{2 b d} .
\end{aligned}
$$

Notice that with this choice the coincidence planes are mapped into the nicely symmetric form:

$$
s+t=0, \quad 1+s=0, \quad 1+t=0 .
$$

These are the boundaries of a right, isosceles triangle with corners at $(s, t)=(-1,-1),(-1,1)$, and $(1,-1)$. The transformation of $\Delta_{\Omega}$ induced by the coordinate change $(u, v)$ to $(s, t)$ is lengthy but straightforward and we do not show it here.

A complete, normalized basis for Lebesgue squareintegrable functions on this domain bounded by Eq. (C4) is provided by the functions $h_{n, m}(s, t)$ :

$$
\begin{aligned}
h_{n, m}(s, t)= & \frac{1}{4}\left(e^{(i \pi / 2)\{-n(s+1)+m(t-1)\}}\right. \\
& -e^{(i \pi / 2)\{-n(s+1)-m(t-1)\}}+e^{(i \pi / 2)\{n(s+1)-m(t-1)\}} \\
& -e^{(i \pi / 2)\{n(s+1)+m(t-1)\}}-e^{(i \pi / 2)\{-m(s+1)+n(t-1)\}} \\
& +e^{(i \pi / 2)\{-m(s+1)-n(t-1)\}}-e^{(i \pi / 2)\{m(s+1)-n(t-1)\}} \\
& \left.+e^{(i \pi / 2)\{m(s+1)+n(t-1)\}}\right), \quad(\mathrm{C} 5)
\end{aligned}
$$

where $n$ and $m$ are positive integers and $n<m$. The matrix elements of the transformed spherical Laplacian can be calculated in this basis and then diagonalized to find the spectrum. In our calculations we set $n, m$ to go up to $N_{\max }=80$. With this upper bound the first 300 energies were quite converged up to the second decimal place. We know this because we did convergence analysis from $60-80$ and found out that the eigenenergies up to the second decimal place were not changing. The calculation time for $N_{\max }=80$ was approximately 10 days on a reasonably powerful desktop computer. If one is interested in higher excited state energies, then one needs to increase $N_{\max }$ in order to get a better precision at the higher end of the spectrum.

Additionally, numerical results are compared to the exact algebraic results for the integrable Coxeter sector for several mass families in $A_{3}, C_{3}$, and $H_{3}$ to confirm the uncertainty estimates. And as we describe in the main text, we compare the level density of the spectrum to the prediction of Weyl's law in order to establish that all eigenstates are found by this method.

[1] S. Albeverio, F. Gesztesy, R. Hoegh-Krohn, and H. Holden, Solvable Models in Quantum Mechanics (Springer-Verlag, Berlin, 1988).

[2] B. Sutherland, Beautiful Models: 70 Years of Exactly Solved Quantum Many-Body Problems (World Scientific Publishing Company, River Edge, NJ, 2004).

[3] M. Gaudin, The Bethe Wavefunction (Cambridge University Press, Cambridge, England, 2014).

[4] E. H. Lieb and W. Liniger, Exact Analysis of an Interacting Bose Gas. I. The General Solution and the Ground State, Phys. Rev. 130, 1605 (1963).

[5] M. Girardeau, Relationship between Systems of Impenetrable Bosons and Fermions in One Dimension, J. Math. Phys. (N.Y.) 1, 516 (1960).

[6] F. Calogero, Solution of the One-Dimensional N-Body Problems with Quadratic and/or Inversely Quadratic Pair Potentials, J. Math. Phys. (N.Y.) 12, 419 (1971).

[7] F. Calogero, Calogero-Moser System, Scholarpedia 3, 7216 (2008).

[8] M. A. Olshanetsky and A. M. Perelomov, Quantum Integrable Systems Related to Lie Algebras, Phys. Rep. 94, 313 (1983).

[9] M. A. Cazalilla, R. Citro, T. Giamarchi, E. Orignac, and M. Rigol, One Dimensional Bosons: From Condensed Matter Systems to Ultracold Gases, Rev. Mod. Phys. 83, 1405 (2011).

[10] X.-W. Guan, M. T. Batchelor, and Chaohong Lee, Fermi Gases in One Dimension: From Bethe Ansatz to Experiments, Rev. Mod. Phys. 85, 1633 (2013).

[11] M. Olshanii, Atomic Scattering in the Presence of an External Confinement and a Gas of Impenetrable Bosons, Phys. Rev. Lett. 81, 938 (1998).

[12] T. Kinoshita, T. Wenger, and D. S. Weiss, A Quantum Newton's Cradle, Nature (London) 440, 900 (2006).

[13] F. Serwane, G. Zürn, T. Lompe, T. B. Ottenstein, A. N. Wenz, and S. Jochim, Deterministic Preparation of a Tunable Few-Fermion System, Science 332, 336 (2011).

[14] A. N. Wenz, G. Zürn, S. Murmann, I. Brouzos, T. Lompe, and S. Jochim, From Few to Many: Observing the Formation of a Fermi Sea One Atom at a Time, Science 342, 457 (2013).

[15] D. P. DiVincenzo, D. Bacon, J. Kempe, G. Burkard, and K. B. Whaley, Universal Quantum Computation with the Exchange Interaction, Nature (London) 408, 339 (2000).

[16] S. Bose, Quantum Communication through an Unmodulated Spin Chain, Phys. Rev. Lett. 91, 207901 (2003). 
[17] A. G. Volosniev, D. Petrosyan, M. Valiente, D. V. Fedorov, A. S. Jensen, and N. T. Zinner, Engineering the Dynamics of Effective Spin-Chain Models for Strongly Interacting Atomic Gases, Phys. Rev. A 91, 023620 (2015).

[18] An exception is the free-space CSM model with mass-scaled interaction strengths in Ref. [19].

[19] D. Sen, A Multispecies Calogero-Sutherland Model, Nucl. Phys. B479, 554 (1996).

[20] O. Bohigas, in Chaos and Quantum Physics, Proceedings of the Les Houches Summer School, Session No. LII, edited by M.-J. Gianoni, A. Voros, and J. Zinn-Justin (North-Holland, Amsterdam, 1991).

[21] C. B. Whan, Hierarchical Level-Clustering in TwoDimensional Harmonic Oscillators, Phys. Rev. E 55, R3813 (1997).

[22] I. Brouzos and A. Foerster, Trace of Broken Integrability in Stationary Correlation Properties, Phys. Rev. A 89, 053623 (2014).

[23] M. Rigol, V. Dunjko, and M. Olshanii, Thermalization and Its Mechanism for Generic Isolated Quantum Systems, Nature (London) 452, 854 (2008).

[24] J. De Nardis, B. Wouters, M. Brockmann, and J.-S. Caux, Solution for an Interaction Quench in the Lieb-Liniger Bose Gas, Phys. Rev. A 89, 033601 (2014).

[25] C. Neill et al., Ergodic Dynamics and Thermalization in an Isolated Quantum System, Nat. Phys. 12, 1037 (2016).

[26] N. P. Mehta, Born-Oppenheimer Study of Two-Component Few-Particle Systems Under One-Dimensional Confinement, Phys. Rev. A 89, 052706 (2014); , Erratum Phys. Rev. A, 92, 029901(E) (2015).

[27] A. S. Dehkharghani, A. G. Volosniev, and N. T. Zinner, Quantum Impurity in a One-Dimensional Trapped Bose Gas, Phys. Rev. A 92, 031601 (2015).

[28] D. Pęcak, M. Gajda, and T. Sowiński, Two-Flavour Mixture of a Few Fermions of Different Mass in a One-Dimensional Harmonic Trap, New J. Phys. 18, 013030 (2016).

[29] N. P. Mehta and C. D. Morehead, Few-Boson Processes in the Presence of an Attractive Impurity Under One-Dimensional Confinement, Phys. Rev. A 92, 043616 (2015).

[30] D. Pęcak and T. Sowiński, Few Strongly Interacting Ultracold Fermions in One-Dimensional Traps of Different Shapes, Phys. Rev. A 94, 042118 (2016).

[31] A. S. Dehkharghani, A. G. Volosniev, and N. T. Zinner, Impenetrable Mass-Imbalanced Particles in One-Dimensional Harmonic Traps, J. Phys. B 49, 085301 (2016).

[32] D. Pęcak, M. Gajda, and T. Sowiński, Experimentally Accessible Invariants Encoded in Interparticle Correlations of Harmonically Trapped Ultra-Cold Few-Fermion Mixtures, arXiv: 1703.08116.

[33] D. Pęcak, A. S. Dehkharghani, N. T. Zinner, and T. Sowiński, Four Fermions in a One-Dimensional Harmonic Trap: Accuracy of a Variational-Ansatz Approach, Phys. Rev. A 95, 053632 (2017).

[34] M. Olshanii and S. G. Jackson, An Exactly Solvable Quantum Four-Body Problem Associated with the Symmetries of an Octacube, New J. Phys. 17, 105005 (2015).

[35] M. Olshanii, T. Scoquart, J. Seaward, and S. G. Jackson, Exactly Solvable Quantum Few-Body Systems Associated with the Symmetries of the Three-Dimensional and FourDimensional Icosahedra, SciPost Phys. 1, 005 (2016).
[36] Maxim Olshanii, Thibault Scoquart, Dmitry Yampolsky, Vanja Dunjko, and Steven Glenn Jackson, Creating Entanglement Using Integrals of Motion, arXiv:1610.01060.

[37] A. G. Volosniev, D. V. Fedorov, A. S. Jensen, M. Valiente, and N. T. Zinner, Strongly Interacting Confined Quantum Systems in One Dimension, Nat. Commun. 5, 5300 (2014).

[38] F. Deuretzbacher, D. Becker, J. Bjerlin, S. M. Reimann, and L. Santos, Quantum Magnetism without Lattices in Strongly Interacting One-Dimensional Spinor Gases, Phys. Rev. A 90, 013611 (2014).

[39] N. L. Harshman, One-Dimensional Traps, Two-Body Interactions, Few-Body Symmetries. II. N Particles, Few-Body Syst. 57, 45 (2016).

[40] D. Roscher, J. Braun, J.-W. Chen, and J. E. Drut, Fermi Gases with Imaginary Mass Imbalance and the Sign Problem in Monte-Carlo Calculations, J. Phys. G 41, 055110 (2014).

[41] H. S. M. Coxeter, Regular Polytopes, 3rd ed. (Dover Publications, New York, 1973).

[42] K. G. Boreskov, A. V. Turbiner, and J. C. L. Vieyra, Solvability of the Hamiltonians Related to Exceptional Root Spaces: Rational Case, Commun. Math. Phys. 260, 17 (2005).

[43] M. A. G. García and A. V. Turbiner, The Quantum $\mathrm{H}_{3}$ Integrable System, Int. J. Mod. Phys. A 25, 5567 (2010).

[44] M. A. G. García and A. V. Turbiner, The Quantum $H_{4}$ Integrable System, Mod. Phys. Lett. A 26, 433 (2011).

[45] P. Tempesta, A. V. Turbiner, and P. Winternitz, Exact Solvability of Superintegrable Systems, J. Math. Phys. (N.Y.) 42, 4248 (2001).

[46] S. Post, S. Tsujimoto, and L. Vinet, Families of Superintegrable Hamiltonians Constructed from Exceptional Polynomials, J. Phys. A 45, 405202 (2012).

[47] J. B. McGuire, Study of Exactly Soluble One-Dimensional N-Body Problems, J. Math. Phys. (N.Y.) 5, 622 (1964).

[48] B. Sutherland, Nondiffractive Scattering: Scattering from Kaleidoscopes, J. Math. Phys. (N.Y.) 21, 1770 (1980).

[49] A. Lamacraft, Diffractive Scattering of Three Particles in One Dimension: A Simple Result for Weak Violations of the Yang-Baxter Equation, Phys. Rev. A 87, 012707 (2013).

[50] J.-S. Caux and J. Mossel, Remarks on the Notion of Quantum Integrability, J. Stat. Mech. (2011) P02023.

[51] N. W. Evans, Superintegrability in Classical Mechanics, Phys. Rev. A 41, 5666 (1990).

[52] S. Wojciechowski, Superintegrability of the CalogeroMoser System, Phys. Lett. A 95, 279 (1983).

[53] T. Hakobyan, O. Lechtenfeld, and A. Nersessian, Superintegrability of Generalized Calogero Models with Oscillator or Coulomb Potential, Phys. Rev. D 90, 101701 (2014).

[54] G. Casati and J. Ford, Computer Study of Ergodicity and Mixing in a Two-Particle, Hard Point Gas System, J. Comput. Phys. 20, 97 (1976).

[55] P. J. Richens and M. V. Berry, Pseudointegrable Systems in Classical and Quantum Mechanics, Physica D (Amsterdam) 2D, 495 (1981).

[56] E. Gutkin, Billiards in Polygons: Survey of Recent Results, J. Stat. Phys., 83, 7 (1996).

[57] S. L. Glashow and L. Mittag, Three Rods on a Ring and the Triangular Billiard, J. Stat. Phys. 87, 937 (1997). 
[58] R. Artuso, G. Casati, and I. Guarneri, Numerical Study on Ergodic Properties of Triangular Billiards, Phys. Rev. E 55, 6384 (1997).

[59] G. Casati and T. Prosen, Mixing Property of Triangular Billiards, Phys. Rev. Lett. 83, 4729 (1999).

[60] J. B. McGuire and C. Dirk, Extending the Bethe Ansatz: The Quantum Three-Particle Ring, J. Stat. Phys. 102, 971 (2001).

[61] T. Araújo Lima, S. Rodríguez-Pérez, and F. M. de Aguiar, Ergodicity and Quantum Correlations in Irrational Triangular Billiards, Phys. Rev. E 87, 062902 (2013).

[62] J. Wang, G. Casati, and T. Prosen, Nonergodicity and Localization of Invariant Measure for Two Colliding Masses, Phys. Rev. E 89, 042918 (2014).

[63] J. E. Humphreys, Reflection Groups and Coxeter Groups (Cambridge University Press, Cambridge, England, 1992).

[64] R. Goodman, Alice through Looking Glass after Looking Glass: The Mathematics of Mirrors and Kaleidoscopes, Am. Math. Mon. 111, 281 (2004).

[65] V. I. Yukalov and M. D. Girardeau, Fermi-Bose Mapping for One-Dimensional Bose Gases, Laser Phys. Lett. 2, 375 (2005).

[66] N. L. Harshman, Spectroscopy for a Few Atoms Harmonically Trapped in One Dimension, Phys. Rev. A 89, 033633 (2014).

[67] L. Brink, T. H. Hansson, and M. A. Vasiliev, Explicit Solution to the N-Body Calogero Problem, Phys. Lett. B 286, 109 (1992).

[68] K. Vacek, A. Okiji, and N. Kawakami, Eigenfunctions for $\mathrm{SU}(\nu)$ Particles with $1 / r^{2}$ Interaction in Harmonic Confinement, J. Phys. A 27, L201 (1994).

[69] J.S. Avery, Hyperspherical Harmonics: Applications in Quantum Theory (Springer, Dordrecht, 1989).

[70] R. J. Yáñez, W. Van Assche, and J. S. Dehesa, Position and Momentum Information Entropies of the D-Dimensional Harmonic Oscillator and Hydrogen Atom, Phys. Rev. A 50, 3065 (1994).

[71] V. Ivrii, 100 Years of Weyl's Law, Bull. Math. Sci. 6, 379 (2016).

[72] J. Brooks and J. Strantzen, Spherical Triangles of Area $\pi$ and Isosceles Tetrahedra, Math. Mag. 78, 311 (2005).

[73] E. W. Weisstein, Spherical Trigonometry. From Wolfram MathWorld-A Wolfram Web Resource, http://mathworld .wolfram.com/SphericalTrigonometry.html.

[74] A. Hobson, Ergodic Properties of a Particle Moving Elastically Inside a Polygon, J. Math. Phys. (N.Y.) 16, 2210 (1975).

[75] M. L. Mehta, Basic Sets of Invariant Polynomials for Finite Reflection Groups, Communications in Algebra 16, 1083 (1988).
[76] A. Saghatelian, Constants of Motion of the Four-Particle Calogero Model, Phys. At. Nucl. 75, 1288 (2012).

[77] C. Kittel, Introduction to Solid State Physics, 8th ed. (Wiley, Hoboken, NJ, 2004).

[78] B. Gadway, D. Pertot, J. Reeves, M. Vogt, and D. Schneble, Glassy Behavior in a Binary Atomic Mixture, Phys. Rev. Lett. 107, 145306 (2011).

[79] L. Khaykovich, F. Schreck, G. Ferrari, T. Bourdel, J. Cubizolles, L. D. Carr, Y. Castin, and C. Salomon, Formation of a Matter-Wave Bright Soliton, Science 296, 1290 (2002).

[80] K. E. Strecker, G. B. Partridge, A. G. Truscott, and R. G. Hulet, Formation and Propagation of Matter-Wave Soliton Trains, Nature (London) 417, 150 (2002).

[81] S. L. Cornish, S. T. Thompson, and C. E. Wieman, Formation of Bright Matter-Wave Solitons during the Collapse of Attractive Bose-Einstein Condensates, Phys. Rev. Lett. 96, 170401 (2006).

[82] R. G. Hulet (private communication).

[83] We note that in principle the relative Hamiltonian for any of the $N=4$ families also could be realized with a noninteracting Bose-Einstein condensate in a spherically symmetric harmonic trap sliced by six sheets of intense laser light, or even just three sheets to create a sector.

[84] J. M. Deutsch, Quantum Statistical Mechanics in a Closed System, Phys. Rev. A 43, 2046 (1991).

[85] M. Srednicki, Chaos and Quantum Thermalization, Phys. Rev. E 50, 888 (1994).

[86] Z. Zhang, V. Dunjko, and M. Olshanii, Atom Transistor from the Point of View of Nonequilibrium Dynamics, New J. Phys. 17, 125008 (2015).

[87] V. A. Yurovsky and M. Olshanii, Memory of the Initial Conditions in an Incompletely Chaotic Quantum System: Universal Predictions with Application to Cold Atoms, Phys. Rev. Lett. 106, 025303 (2011).

[88] N. Friedman, A. Kaplan, D. Carasso, and N. Davidson, Observation of Chaotic and Regular Dynamics in AtomOptics Billiards, Phys. Rev. Lett. 86, 1518 (2001).

[89] F. Werner and Y. Castin, Unitary Gas in an Isotropic Harmonic Trap: Symmetry Properties and Applications, Phys. Rev. A 74, 053604 (2006).

[90] M. Hamermesh, Group Theory and Its Application to Physical Problems, (Dover Publications, New York, 1989).

[91] B. G. Wybourne, Classical Groups for Physicists, 1st ed. (John Wiley \& Sons Inc, New York, 1974).

[92] M. Harmer, The Spectra of the Spherical and Euclidean Triangle Groups, J. Aust. Math. Soc. 84, 217 (2008).

[93] S. T. Rittenhouse, J. von Stecher, J. P. D’Incao, N. P. Mehta, and C.H. Greene, The Hyperspherical Four-Fermion Problem, J. Phys. B 44, 172001 (2011). 